\title{
Anatomic vs. non-anatomic liver resection for hepatocellular carcinoma: standard of care or unfilled promises?
}

\author{
Nicole M. Nevarez, Adam C. Yopp \\ Department of Surgery, University of Texas Southwestern Medical Center, Dallas, Texas 75390, USA. \\ Correspondence to: Dr. Adam C. Yopp, Department of Surgery, University of Texas Southwestern Medical Center, 5323 Harry \\ Hines Boulevard, Dallas, Texas 75390, USA. E-mail: adam.yopp@utsouthwestern.edu
}

How to cite this article: Nevarez NM, Yopp AC. Anatomic vs. non-anatomic liver resection for hepatocellular carcinoma: standard of care or unfilled promises? Hepatoma Res 2021;7:66. https://dx.doi.org/10.20517/2394-5079.2021.66

Received: 24 May 2021 First Decision: 28 Jun 2021 Revised: 5 Jul 2021 Accepted: 27 Jul 2021 Published: 15 Oct 2021

Academic Editor: Georgios Tsoulfas Copy Editor: Yue-Yue Zhang Production Editor: Yue-Yue Zhang

\begin{abstract}
Hepatocellular carcinoma (HCC) is one of the leading causes of cancer-related death not only in the United States but in the world. One of the curative treatment options for early-stage HCC is surgical resection, which can be divided into two approaches: anatomic and nonanatomic. The theoretical advantage of anatomic liver resection is excising the entire primary tumor along with adjacent liver parenchyma containing micrometastases that reside in the surrounding portal tributaries. However, the superiority of anatomic vs. nonanatomic liver resection in patients with HCC is controversial. While this is a feasible strategy for patients with preserved liver function, it may not be ideal for patients with cirrhosis, who rely on parenchymal-sparing or nonanatomic approaches to maximize their future liver remnant and prevent post-operative liver failure. This review identifies and critically analyzes the evidence for anatomic vs. nonanatomic liver resection for HCC.
\end{abstract}

Keywords: Hepatocellular carcinoma, anatomic liver resection, parenchymal-sparing liver resection, liver surgery

\section{INTRODUCTION}

Hepatocellular carcinoma (HCC) has the 6th highest incidence in the world and is responsible for the third most common cause of cancer-related mortality ${ }^{[1,2]}$. Typically found in patients with known chronic liver disease with concomitant cirrhosis, overall prognosis following HCC diagnosis depends on factors that 
include tumor stage, patient functional status, and degree of hepatic dysfunction. A concomitant varied cancer diagnosis and degree of liver dysfunction provide heterogeneity in HCC presentation leading to the need for multidisciplinary treatment modalities ${ }^{[3]}$. The only curative therapies for HCC treatment are partial hepatectomy, hepatic transplantation, or tumor ablation. Despite five-year survival rates following surgical resection near $60 \%$, less than $40 \%$ of patients newly diagnosed with HCC are suitable for partial hepatectomy due to advanced tumor biology and low HCC screening rates ${ }^{[4-7]}$.

Careful patient selection, advances in perioperative care, and post-operative care are associated with decreased 30-day morbidity and mortality following partial hepatectomy for HCC over the past three decades. As more patients are undergoing surgical resection for HCC, it is paramount to investigate surgical methods to reduce locoregional rates of recurrence that approach nearly $50 \%$ within five years following surgery. One mitigating variable controlled during partial hepatectomy is obtaining tumor-free or negative resection margins but still preserving sufficient liver parenchyma to decrease rates of post-hepatectomy liver failure. There has evolved two surgical techniques of performing curative resection for HCC as such. The first is anatomic resection (AR), whereby the tumor-free margin is dependent on segmental liver anatomy and not cut liver surface margin. The second is non-anatomic resection (NAR), whereby parenchymal preservation is paramount, and tumor-free margin is contingent on the cut liver surface.

Despite AR being described nearly three decades ago and based on theoretical reduction of micrometastatic disease, its use during partial hepatectomy for HCC is not used worldwide. The goals of this review are to describe the surgical evolution of AR as it relates to partial hepatectomy, discuss the hypothetical reasonings explaining the benefits of AR during hepatic resection for HCC, and to elucidate if AR is superior to NAR and thus should be considered standard of care during partial hepatectomy for HCC or worthy of future clinical trials.

\section{RATIONALE FOR ANATOMIC RESECTION IN HCC}

Theoretically, utilization of AR during partial hepatectomy in HCC patients should eradicate intrahepatic metastases by removing tumor-bearing portal tributaries ${ }^{[8]}$. The rate of recurrence following partial hepatectomy for HCC approaches $70 \%$ over a five-year post-operative period and has a bimodal distribution of recurrence based on intrahepatic metastasis and de novo HCC development secondary to chronic liver diseas $\mathrm{e}^{[9,10]}$. Within the first two years following partial hepatectomy, the vast majority of recurrent HCC tumors are secondary to intrahepatic metastasis from the primary tumor. Clonality studies of both primary and recurrent HCC tumors demonstrate that these early recurrences are associated with poor tumor biology, including advanced tumor grade, microsatellitosis, and vascular invasion. HCC recurrences following two years after partial hepatectomy are more likely related to the underlying chronic liver disease, and the tumors are consistent with non-overt poor tumor biology $\mathrm{y}^{[1,12]}$.

Early HCC recurrences following partial hepatectomy are based upon the hypothesis that HCC has a distinct predilection for vascular invasion through the portal venous system. The dissemination of HCC tumor cells into neighboring portal veins allows the formation of micro tumor thrombi and subsequent tumor satellite formation throughout the liver manifesting as recurrent HCC tumors ${ }^{[9]}$. Thus, in theory, AR hinders this dissemination, reducing tumor cell flow throughout the liver and reducing HCC recurrences in the early period following partial hepatectomy. Unfortunately, this working theory is predisposed on the assumption that tumor cells are within the neighboring portal venous system and have not already disseminated throughout the liver as tumor circulating cells prior to resection. Disputing the theoretical advantage of AR, Sun et al. ${ }^{[13]}$ reported that the percentages of circulating tumor cells in blood sampled from a peripheral vein, peripheral artery, hepatic vein, inferior vena cava, and portal vein prior to HCC resection 
were $69 \%, 45 \%, 81 \%, 40 \%$, and $59 \%$, respectively.

\section{ANATOMIC RESECTION AND ASSOCIATED SURGICAL TECHNIQUES}

AR, first described by Professor Masatoshi Makuuchi in 1985, is characterized as resection of the primary HCC tumor in addition to the hepatic segment or subsegment wherein the tumor resides, thereby resecting both the appropriate hepatic venous drainage and associated portal venous and hepatic arterial blood supply. NAR usually involving a less extensive parenchymal resection, or parenchymal-sparing, is predicated on obtaining grossly negative surgical margins and is not based on the drainage and blood supply of the anatomical location of the tumor.

AR can be completed using two of the following techniques: (1) ultrasonically guided transection ${ }^{[14]}$ or (2) Glissonean pedicle transection, anatomically relying on either segmentectomy or sectionectomy, including bisectionectomy, hemihepatectomy, or trisectionectomy.

\section{Ultrasonographic guided transection technique}

In 1985, Makuuchi et al ${ }^{[15]}$ first described what is now known as positive staining by infusing indigo carmine dye into the neighboring portal venous structure of the hepatic parenchyma wherein the HCC tumor resides to allow identification of landmarks to guide a targeted approach during hepatic resection of a segmental or subsegmental area. Later reports described a modification of the Makuuchi approach through indocyanine green (ICG) fluorescence ${ }^{[16,17]}$. In both techniques, the hepatic artery is compressed proximally near the hepatic hilus, and either indigo carmine or ICG is injected under IOUS guidance into the appropriate portal venous tributaries. Parenchymal transection is then completed by proceeding outwards from the hepatic parenchymal surface then inwards to the portal pedicle using the intensity of the indigo carmine or ICG dye within the liver parenchyma as a guide. This parenchymal transection is completed corresponding to the target area of the liver with the tumor, preserving the appropriate main hepatic vein, ligating the portal pedicle proximally ${ }^{[18]}$.

Utilization of IOUS guided injection of either ICG or indigo carmine to perform AR requires the operating surgeon to have advanced ultrasonography techniques and to accurately occlude the appropriate arterial inflow to ensure reliable dye infusion and avoid dye reflux in non-tumor portal branches. Unreliable positive dye staining through the IOUS technique ranges from $40 \%$ to $58 \%^{[16,17,19]}$. Utilization of a fluorescence imaging system in combination with finger compression of the appropriate anatomical segments may address the concerns of unreliable staining ${ }^{[20]}$.

Additionally, the usage of ICG can also be used for negative staining ${ }^{[21]}$. Unlike positive staining, ICG is injected peripherally and intravenously. Also, unlike positive staining, where the hepatic artery is compressed during injection, the portal pedicle of the targeted hepatic segment with HCC tumor is compressed. All the hepatic parenchyma will fluoresce except the segment with the portal pedicle clamped. Negative staining is particularly valuable in minimally invasive surgical approaches. While positive staining requires adeptness with IOUS for injection in portal venous tributaries, negative staining has the advantage of being administered intravenously and compresses vessels (i.e., portal pedicles) that are easily accessible in most hepatic segments.

\section{Glissonean pedicle transection technique}

Three main approaches to portal pedicle inflow control at the hepatic hilum have been described: extrafascial, intrafascial, and combination extrafascial/transfissural. Couinaud ${ }^{[22]}$ first described an extrafascial approach to perform an anatomical left hepatectomy in 1985. This approach was further 
modified by Takasaki et al. ${ }^{[23,24]}$ in 1986 in their description of a hepatic resection to include dividing the hepatic parenchyma into three distinct subsegments: right, middle, and left (the caudate lobe was initially not included). The division of the liver into four segments (including the caudate) was based upon cadaveric studies that demonstrated that connective tissue or peritoneum sheaths the hepatic artery, portal vein, and biliary structures within the hepatoduodenal ligament [Figure 1]. Like the trunk of a tree, the main Glissonean pedicle further divides at the hepatic hilus as right and left primary branches, subdividing further into secondary branches (right and middle/left, respectively). The right secondary branch entails hepatic segments 6 and 7, and the middle secondary branch entailing segments 5 and 8 , with the right hepatic vein occupying the area between the middle and right secondary branches. The left secondary branch entails segments 2,3 , and 4 , with the middle hepatic vein lying between the middle and left secondary branches. This division of the liver into branches and segments utilizes the Brisbane terminology ${ }^{[25]}$. Takasaki et al. ${ }^{[24]}$ further described subdividing each of the three secondary branches into tertiary 6-8 smaller areas or "cone units".

Resection of HCC tumors utilizing the Takasaki modification of the extrafascial approach involves first dividing the associated Glissonean pedicle segmental branch and then proceeding with a dissection within the intersegmental anatomical plane of the accompanying hepatic vein. This allows resection of the hepatic parenchyma but the preservation of the corresponding hepatic vein.

Resection of "cone units" within the tumor parenchymal segment can be carried out by defining tertiary branches and utilizing a hilar approach for parenchymal dissection. This technique is useful when HCC tumors are within limited parenchymal anatomical spaces.

Although the parenchymal sparing approach of AR via a Glissonean approach is appealing, its use in resecting lesions, especially in "cone unit" areas in segments 7 or 8 , is less than ideal due to the wide variability of tertiary branches within these Couinaud segments. For example, in segments 7 or 8 , four or more tertiary branches, in cadaveric studies, have been described to supply one "cone unit". Furthermore, attempting to isolate sub-segmental tertiary branches of portal veins and hepatic arteries, especially in segments 7 and 8 , potentially carries the risk of non-parenchymal dissection outside the planned parenchymal plane leading to a non-parenchymal sparing approach.

If HCC tumors are located in an area where either a right or left hepatectomy is warranted, utilization of the Glissonean pedicle approach is straightforward, relying on standard extrahepatic pedicle landmarks. However, if the tumor is positioned away from the hepatic hilus, for example, in segments 7 or 8 , the IOUS guided technique with dye localization appears to be easier to perform, especially with the adeptness of IOUS.

Due to anatomical variability within the liver, surgeons must be prepared to utilize both the ultrasonically guided or Glissonean pedicle transection approach based on preoperative contrast-enhanced imaging to ensure AR is completed in a safe manner with few intraoperative complications. In addition, since both approaches rely on a hepatic parenchymal transection plane along major hepatic veins, the potential for catastrophic bleeding remains high, and low central venous pressure anesthetic techniques are crucial in performing AR for HCC tumor resection safely.

\section{ONCOLOGICAL OUTCOMES COMPARING ANATOMIC VS . NON-ANATOMIC: THE EVIDENCE}

As mentioned above, theoretically, AR in patients with HCC should offer a survival benefit as long-term 


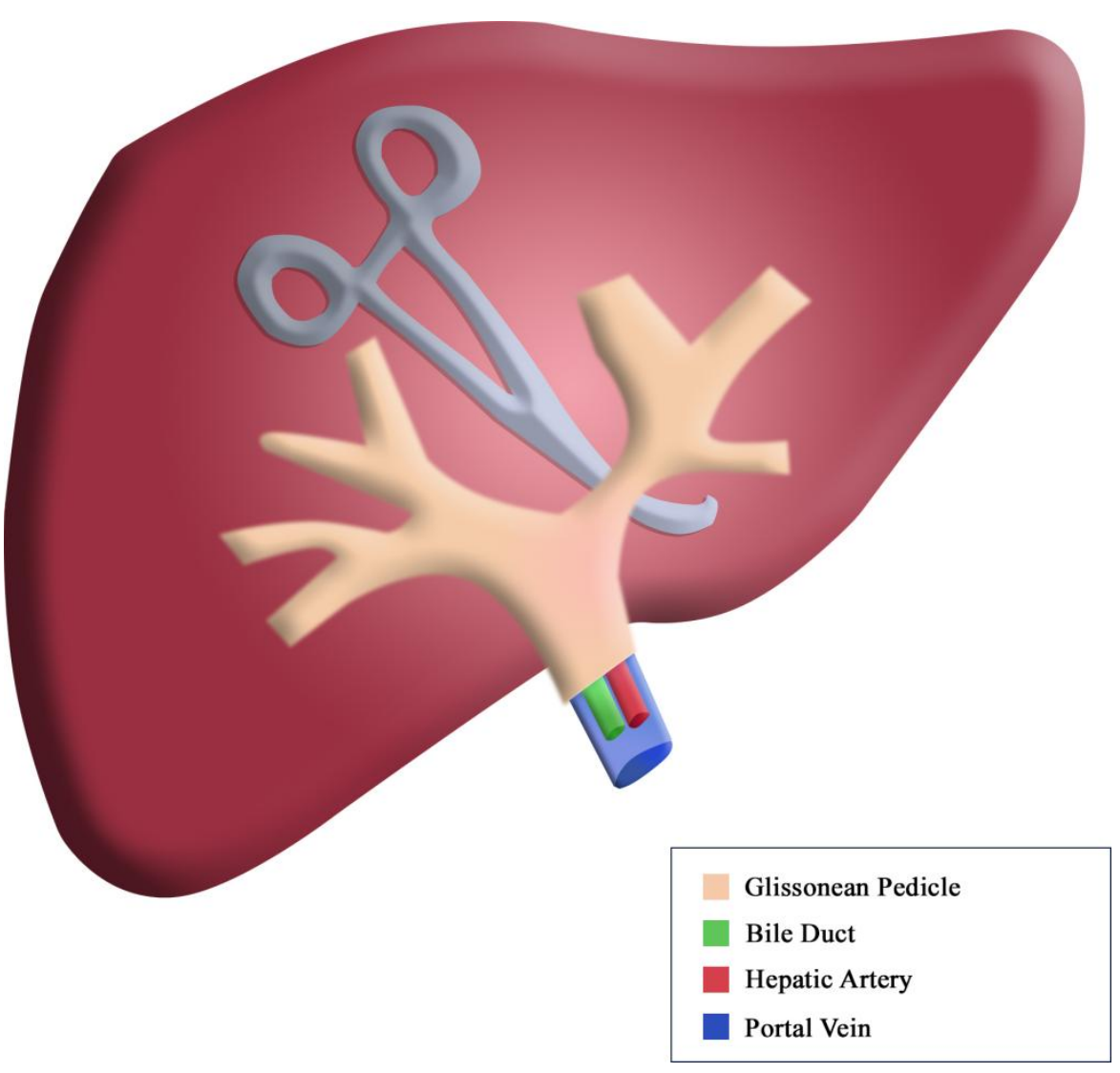

Figure 1. Schematic of a Glissonean pedicle.

oncological outcomes are reflective of time to recurrence and rates of recurrence as a surrogate of tumor biology. However, there is an alarming paucity of published data comparing the two surgical approaches with reliance on largely retrospective, single institutional studies with varying definitions of what constitutes an anatomical hepatic resection and inherent institutional bias for the predilection of AR $v s$. NAR as the preferred resection technique.

Currently, there is only one randomized controlled trial comparing AR and NAR approaches for HCC tumor resection with regards to differences in perioperative and oncological outcomes. Feng et al. ${ }^{[26]}$, in 2017, described a single institutional clinical trial with 105 HCC patients who were randomized to either AR or NAR. The primary endpoint of this prospective study was a two-year local recurrence rate, defined as a recurrence in the same hepatic section as the resected tumor. A significantly decreased two-year local recurrence rate was demonstrated with $\mathrm{AR}$ compared to NAR ( $30 \% v s .59 \%, P=0.001$, respectively). However, neither overall recurrence rate nor overall survival was improved in the cohort of patients undergoing AR. Due to concerns of an association of increased post-operative complications following AR, the authors examined 30-day perioperative or post-operative complication rates between AR and NAR. There were no significant differences in complication rates between AR and NAR. Limitations include the use of local recurrence within the resected section as the primary endpoint, as recurrences either extrahepatically or in other areas of the liver were not considered events. This censoring of non-segmental recurrences is a key determinant behind the lack of significance in recurrence-free survival between the two groups. The local recurrence rate is too narrow of a primary endpoint, as theoretically, the rationale of AR is postulated on the theory of reduction in micrometastatic satellitosis or cells found in nearby portal venous 
tributaries. There are also concerns regarding the widespread applicability of the study results in patients with non-hepatitis B-related etiology of chronic liver disease.

Since there is a paucity of randomized controlled studies, studies contrasting AR and NAR have largely relied on retrospective case series from single institutions with small volumes of patients and a heterogenous patient population limiting the generalizability of study findings. As such, multiple systemic reviews and meta-analyses have been conducted in an attempt to collate the patient data and reach a consensus about AR vs. NAR [Table 1] ${ }^{[27-33]}$. Surprisingly, although many of the studies include cases obtained from otherwise similar retrospective studies, conclusions vary between the studies depending on the variables and outcome measures that were included in the meta-analyses.

In 2011, Zhou et al. ${ }^{[27]}$ preformed a meta-analysis of 16 retrospective studies published between 1996 and 2010. Two thousand nine hundred and seventeen total patients (AR: 1577 and NAR: 1340) met the inclusion criteria. The primary outcomes were three- and five-year overall and disease-free survival (DFS) and local intrahepatic recurrence rates. Secondary outcomes were surgical morbidity and mortality. A significantly higher five-year overall survival (OS) $(66.8 \%$ vs. 55.5\% respectively, $P=0.006)$ was seen in patients undergoing AR. In addition, AR was associated with reduced local intrahepatic recurrence rates (6.9\% vs. $22.4 \%$, respectively). DFS rates in the NAR cohort were increased compared to the AR group at both 3 years $(34.5 \%$ vs. $52.1 \%)$ and 5 years $(43.9 \%$ vs. $25.3 \%)$. There were no significant differences in perioperative mortality or morbidity rates between AR and NAR.

In 2012, Cucchetti et al. ${ }^{[28]}$ included many of the same studies as Zhou et al. ${ }^{[27]}$ in their meta-analysis with systemic review comparing AR and NAR in HCC patients. As expected, both the five-year overall survival and DFS were similar to the previous study, with NAR associated with worse outcome measures. In addition, post-operative morbidity rates were similar between the two operative approaches. The authors, furthermore, utilized a meta-regression technique incorporating covariates of the 18 included studies to associate both overall and disease-free survival rates by the degree of liver dysfunction. The NAR cohort included a higher prevalence of cirrhosis and hepatitis $\mathrm{C}$ virus (HCV), and increased hepatic dysfunction than the AR cohort, using cirrhosis as a measure of liver dysfunction. Each of these individual covariates has been demonstrated to be associated with increased rates of local and non-local rates of recurrence ${ }^{[12,32,33]}$. The use of a meta-regression and inclusion of the presence of cirrhosis as a mitigating covariate of both five-year overall survival and DFS reduces the residual heterogeneity; thus, neither AR nor NAR was associated with favorable outcomes.

In 2018, Moris et al.$^{[31]}$ incorporated data from 43 studies involving 12,429 patients undergoing either AR or NAR for HCC. Patients in the AR cohort had decreased rates of HCV as underlying etiology of liver disease, larger tumor size, higher intraoperative blood loss, longer operative time, and wider margins. Similar to previous meta-analyses, no statistically significant difference in morbidity and mortality was identified between the AR and NAR groups. Also, similar to prior studies, the five-year OS and DFS rates were significantly higher in the AR group compared to the NAR group. Interestingly, similar to the study by Cucchetti et al..$^{[28]}$, in a separate sub-analysis just including patients with known cirrhosis, the five-year OS and DFS differences seen between the two groups were no longer evident. Furthermore, no significant differences were seen in the overall or early recurrence rate.

A prominent limitation of the meta-analyses mentioned above is the heterogeneity of the patient populations within the studies. Many of the study populations are vastly different in their patient characteristics, such as categorization of underlying cirrhosis, Child's classification, HCV serostatus, and 
Table 1. Summary of outcomes following anatomic resection vs. nonanatomic resection

\begin{tabular}{|c|c|c|c|c|}
\hline Ref. & Year & Classification & Number of patients & Outcome \\
\hline Feng et al. ${ }^{[26]}$ & 2017 & $\begin{array}{l}\text { AR } \\
\text { NAR }\end{array}$ & $\begin{array}{l}52 \\
53\end{array}$ & $\begin{array}{l}\text { Median OS: } 210 \text { weeks } \\
150 \text { weeks }\end{array}$ \\
\hline Zhou et al. ${ }^{[27]}$ & 2011 & $\begin{array}{l}\text { AR } \\
\text { NAR }\end{array}$ & $\begin{array}{l}1577 \\
1340\end{array}$ & $\begin{array}{l}\text { Five-year OS: } 66.8 \% \\
55.5 \%\end{array}$ \\
\hline Cucchetti et al. ${ }^{[28]}$ & 2012 & $\begin{array}{l}\text { AR } \\
\text { NAR }\end{array}$ & $\begin{array}{l}4012 \\
5024\end{array}$ & $\begin{array}{l}\text { Pooled five-year OS: } 62.0 \% \\
54.4 \%\end{array}$ \\
\hline Huang et al. ${ }^{[29]}$ & 2017 & $\begin{array}{l}\text { AR } \\
\text { NAR }\end{array}$ & $\begin{array}{l}1626 \\
1503\end{array}$ & $\begin{array}{l}\text { Five-year OS: } 69 \% \\
56 \%\end{array}$ \\
\hline Tan et al. ${ }^{[30]}$ & 2017 & $\begin{array}{l}\text { AR } \\
\text { NAR }\end{array}$ & $\begin{array}{l}4576 \\
5640\end{array}$ & $\begin{array}{l}\text { Five-year OS: } 64.9 \% \\
61.1 \%\end{array}$ \\
\hline Moris et al. ${ }^{[31]}$ & 2018 & $\begin{array}{l}\text { AR } \\
\text { NAR }\end{array}$ & $\begin{array}{l}6839 \\
5590\end{array}$ &  \\
\hline Ye et al. ${ }^{[32]}$ & 2012 & $\begin{array}{l}\text { AR } \\
\text { NAR }\end{array}$ & $\begin{array}{l}810 \\
766\end{array}$ & Five-year OS OR: 1.24 \\
\hline Famularo et al. ${ }^{[33]}$ & 2021 & $\begin{array}{l}\text { AR } \\
\text { NAR }\end{array}$ & $\begin{array}{l}1776 \\
1669\end{array}$ & Five-year OS RR: 0.89 \\
\hline
\end{tabular}

OS: Overall survival; HR: hazard ratio; OR; odds ratio; RR: risk ratio.

inherent tumor biologies such as tumor size and microvascular invasion (MVI). Thus, while the metaanalyses draw generalities about the superiority of AR over NAR, it is unclear if this is applicable to all patients.

More recent meta-analyses have attempted to correct for the inherent heterogeneity by narrowing the inclusion criteria of the included studies. In 2021, Sun et al. ${ }^{[13]}$ performed a meta-analysis of 12 studies, including a total of 1480 (AR: 659 and NAR: 821) patients examining AR vs. NAR but included only studies that contained HCC patients with MVI. OS and DFS were increased following AR compared to NAR at 1 year $(P=0.037$ and $P=0.001$, respectively), 3 years $(P=0.001$ and $P=0.001$, respectively), and 5 years $(P=$ 0.001 and $P=0.002$, respectively). There was no significant difference in post-operative complications between the AR and NR groups.

Another recent meta-analysis attempted to address concerns about heterogeneity by only including studies with either propensity-matched cohorts or from the solitary randomized controlled trial. Famularo et al. ${ }^{[33]}$ included 3445 patients (AR: 1776 and NAR: 1669) from eleven propensity-matched studies and the solitary randomized controlled trial. Following propensity score matching, patient and tumor characteristics were comparable among studies. In contrast to many of the previous meta-analyses, OS was similar between AR and NAR at 1,3 , and 5 years $(P=0.62, P=0.81, P=0.21$, respectively). DFS was improved in AR at 1 and 3 years $(P=0.02$ both, respectively) but was similar at 5 years $(\mathrm{RR}=0.94$; 95\%CI: $0.87-1.01, P=0.07)$. There were no outcome differences (overall and disease-free survival) between AR and NAR when accounting for microvascular invasion.

\section{SUMMARY}

The goal of curative hepatectomy in patients with HCC is to offer a margin negative tumor resection with associated micro-metastatic foci while preserving as much parenchyma as possible to ensure low rates of post-hepatectomy liver failure. With this have emerged two approaches for liver resection in early-stage HCC: AR and NAR. Unfortunately, despite a multitude of systematic reviews and meta-analyses, the extreme heterogeneity of included patients makes it difficult to form a consensus on which technique should be undertaken in HCC patients. Thus, a prospective, randomized trial with relevant endpoints and 
stratification of patients is perhaps the only way this dilemma will be finally addressed.

\section{DECLARATIONS}

\section{Authors' contributions}

Thoroughly reviewed the literature and participated in drafting and revision of this manuscript:

Nevarez NM, Yopp AC

Provided administrative and material support: Yopp AC

\section{Availability of data and materials}

Not applicable.

\section{Financial support and sponsorship}

1R01MD012565-01 (Yopp AC and Nevarez NM).

\section{Conflicts of interest}

Both authors declared that there are no conflicts of interest.

\section{Ethical approval and consent to participate}

Not applicable.

\section{Consent for publication}

Not applicable.

\section{Copyright}

(c) The Author(s) 2021.

\section{REFERENCES}

1. Sung H, Ferlay J, Siegel RL, et al. Global cancer statistics 2020: globocan estimates of incidence and mortality worldwide for 36 cancers in 185 countries. CA Cancer J Clin 2021;71:209-49. DOI PubMed

2. Siegel RL, Miller KD, Fuchs HE, Jemal A. Cancer statistics, 2021. CA Cancer J Clin 2021;71:7-33. DOI PubMed

3. Yopp AC, Mansour JC, Beg MS, et al. Establishment of a multidisciplinary hepatocellular carcinoma clinic is associated with improved clinical outcome. Ann Surg Oncol 2014;21:1287-95. DOI PubMed PMC

4. Pang TC, Lam VW. Surgical management of hepatocellular carcinoma. World J Hepatol 2015;7:245-52. DOI PubMed PMC

5. Fan ST, Lo CM, Liu CL, et al. Hepatectomy for hepatocellular carcinoma: toward zero hospital deaths. Ann Surg 1999;229:322-30. DOI PubMed PMC

6. Colella G, Bottelli R, De Carlis L, et al. Hepatocellular carcinoma: comparison between liver transplantation, resective surgery, ethanol injection, and chemoembolization. Transpl Int 1998;11 Suppl 1:S193-6. DOI PubMed

7. Fong Y, Sun RL, Jarnagin W, Blumgart LH. An analysis of 412 cases of hepatocellular carcinoma at a Western center. Ann Surg 1999;229:790-9; discussion 799. DOI PubMed PMC

8. Hasegawa K, Kokudo N, Imamura H, et al. Prognostic impact of anatomic resection for hepatocellular carcinoma. Ann Surg 2005;242:252-9. DOI PubMed PMC

9. Marubashi S, Gotoh K, Akita $\mathrm{H}$, et al. Anatomical versus non-anatomical resection for hepatocellular carcinoma. Br $J$ Surg 2015;102:776-84. DOI PubMed

10. Sakon M, Nagano H, Nakamori S, et al. Intrahepatic recurrences of hepatocellular carcinoma after hepatectomy: analysis based on tumor hemodynamics. Arch Surg 2002;137:94-9. DOI PubMed

11. Chan AWH, Zhong J, Berhane S, et al. Development of pre and post-operative models to predict early recurrence of hepatocellular carcinoma after surgical resection. J Hepatol 2018;69:1284-93. DOI PubMed

12. Xu XF, Xing H, Han J, et al. Risk factors, patterns, and outcomes of late recurrence after liver resection for hepatocellular carcinoma: a multicenter study from China. JAMA Surg 2019;154:209-17. DOI PubMed PMC

13. Sun Z, Li Z, Shi XL, He XW, Chen J, Song JH. Anatomic versus non-anatomic resection of hepatocellular carcinoma with microvascular invasion: a systematic review and meta-analysis. Asian J Surg 2021;S1015-9584(21)00130. DOI PubMed

14. Kang KJ, Ahn KS. Anatomical resection of hepatocellular carcinoma: a critical review of the procedure and its benefits on survival. World J Gastroenterol 2017;23:1139-46. DOI PubMed PMC

15. Makuuchi M, Hasegawa H, Yamazaki S. Ultrasonically guided subsegmentectomy. Surg Gynecol Obstet 1985;161:346-50. PubMed

16. Miyata A, Ishizawa T, Tani K, et al. Reappraisal of a dye-staining technique for anatomic hepatectomy by the concomitant use of 
indocyanine green fluorescence imaging. J Am Coll Surg 2015;221:e27-36. DOI PubMed

17. Inoue Y, Arita J, Sakamoto T, Ono Y, Takahashi M, Takahashi Y, Kokudo N, Saiura A. Anatomical liver resections guided by 3dimensional parenchymal staining using fusion indocyanine green fluorescence imaging. Ann Surg 2015;262:105-11. DOI PubMed

18. Makuuchi M. Surgical treatment for HCC - special reference to anatomical resection. Int J Surg 2013;11:S47-S9. DOI PubMed

19. Viganò L, Procopio F, Mimmo A, et al. Oncologic superiority of anatomic resection of hepatocellular carcinoma by ultrasound-guided compression of the portal tributaries compared with nonanatomic resection: an analysis of patients matched for tumor characteristics and liver function. Surgery 2018;164:1006-13. DOI PubMed

20. Procopio F, Torzilli G, Franchi E, et al. Ultrasound-guided anatomical liver resection using a compression technique combined with indocyanine green fluorescence imaging. HPB (Oxford) 2021;23:206-11. DOI PubMed

21. Ishizawa T, Zuker NB, Kokudo N, Gayet B. Positive and negative staining of hepatic segments by use of fluorescent imaging techniques during laparoscopic hepatectomy. Arch Surg 2012;147:393-4. DOI PubMed

22. Couinaud CM. A simplified method for controlled left hepatectomy. Surgery 1985;97:358-61. 9. PubMed

23. Takasaki K, Kobayashi S, Tanaka S, et al. Newly developed systematized hepatectomy by Glissonean pedicle transection method. Syujutsu 1986;40:7-14. DOI

24. Takasaki K. Glissonean pedicle transection method for hepatic resection: a new concept of liver segmentation. J Hepatobiliary Pancreat Surg 1998;5:286-91. DOI PubMed

25. TCotIH-P-B. The Brisbane 2000 terminology of liver anatomy and resections. HPB 2000;3:333-9.

26. Feng X, Su Y, Zheng S, et al. A double blinded prospective randomized trial comparing the effect of anatomic versus non-anatomic resection on hepatocellular carcinoma recurrence. HPB (Oxford) 2017;19:667-74. DOI PubMed

27. Zhou Y, Xu D, Wu L, Li B. Meta-analysis of anatomic resection versus nonanatomic resection for hepatocellular carcinoma. Langenbecks Arch Surg 2011;396:1109-17. DOI PubMed

28. Cucchetti A, Cescon M, Ercolani G, Bigonzi E, Torzilli G, Pinna AD. A comprehensive meta-regression analysis on outcome of anatomic resection versus nonanatomic resection for hepatocellular carcinoma. Ann Surg Oncol 2012;19:3697-705. DOI PubMed

29. Huang X, Lu S. A Meta-analysis comparing the effect of anatomical resection vs. non-anatomical resection on the long-term outcomes for patients undergoing hepatic resection for hepatocellular carcinoma. HPB (Oxford) 2017;19:843-9. DOI PubMed

30. Tan Y, Zhang W, Jiang L, Yang J, Yan L. Efficacy and safety of anatomic resection versus nonanatomic resection in patients with hepatocellular carcinoma: a systemic review and meta-analysis. PLoS One 2017;12:e186930. DOI PubMed PMC

31. Moris D, Tsilimigras DI, Kostakis ID, et al. Anatomic versus non-anatomic resection for hepatocellular carcinoma: a systematic review and meta-analysis. Eur J Surg Oncol 2018;44:927-38. DOI PubMed

32. Ye JZ, Miao ZG, Wu FX, Zhao YN, Ye HH, Li LQ. Recurrence after anatomic resection versus nonanatomic resection for hepatocellular carcinoma: a meta-analysis. Asian Pac J Cancer Prev 2012;13:1771-7. DOI PubMed

33. Famularo S, Ceresoli M, Giani A, et al. Is it just a matter of surgical extension to achieve the cure of hepatocarcinoma? $J$ Gastrointest Surg 2021;25:94-103. DOI PubMed 